\title{
Medication use evaluation for Clostridium difficile infection: a case of super tertiary care hospital in northeastern Thailand
}

\author{
Cheardchai Soontornpas ${ }^{1}$, \\ Piroon Mootsikapun², \\ Ratchadaporn Soontornpas $3^{3^{*}}$ \\ 1 Division of Clinical Pharmacy, Faculty \\ of Pharmaceutical Sciences, KhonKaen \\ University, KhonKaen, Thailand \\ 2 Department of Medicine, Faculty of \\ Medicine, KhonKaen University, KhonKaen, \\ Thailand \\ 3 Department of Pharmacy Service, Srina- \\ garind Hospital, KhonKaen University, \\ KhonKaen, Thailand
}

*Corresponding author:

Ratchadaporn Soontornpas ratwis@kku.ac.th

Keywords:

Antibiotic associated colitis, Antibiotic associated diarrhea, Clostridium difficile infection

Pharmaceutical Sciences Asia @ 2021 by Faculty of Pharmacy, Mahidol University, Thailand is licensed under CCBY-NC-ND 4.0. To view a copy of this license, visit https:// www.creativecommons.org/ licenses/by-nc-nd/4.0/

\begin{abstract}
Clostridium difficile infection (CDI) is a common and serious nosocomial infection worldwide. It is also problem in our hospital, a medical school in Khon Kaen University. Rationale use of antimicrobial in the treatment of CDI is still being questionable. This study aimed to explore the characteristic and evaluate the treatment of CDI in our setting. This retrospective cohort study was performed in 168 CDI patients admitted at Srinagarind Hospital between October 2013 and September 2016. Patient medical records were reviewed for demographics, comorbidities, antimicrobial exposures, treatments and clinical outcomes. Antimicrobial therapy for CDI was then evaluated in accordance with the current CDI guideline. Most CDI patients aged over 50 years $(72.6 \%)$ and $42.8 \%$ aged over 65 years. Common comorbidities in the patients were cardiovascular disease (47.6\%), diabetes mellitus (27.4\%), chronic kidney disease (20.8\%), malignancy (16.7\%), cerebrovascular disease (11.3\%), chronic hepatitis (3.6\%) and chronic obstructive pulmonary disease $(2.4 \%)$. Patients who had taken antimicrobial during hospitalization prior to CDI diagnosis constituted $83.9 \%$ of total study population. Metronidazole, vancomycin and both were initial therapy in $83.9,4.8$ and $11.3 \%$ of CDI, respectively. Appropriateness of CDI treatment in all terms was $44 \%$ and recovery rate was $62 \%$ of assessable cases. Nine patients $(5.4 \%)$ died during hospitalization. In conclusion, most patients had CDI risk factors including elderly, comorbidity, antimicrobial exposure, and prolonged hospital stay. Inappropriate CDI treatment was found in asymptomatic carriers or inappropriate treatment duration. Initial therapy with metronidazole should change to oral vancomycin which was the first-line therapy in the current guidelines.
\end{abstract}

\section{INTRODUCTION}

Antibiotic associated colitis (AAC) or antibiotic associated diarrhea (AAD) is a gastrointestinal infection caused by a sporeforming gram-positive anaerobic bacillus named Clostridium difficile. (C.difficile) infection (CDI) is the most common nosocomial infectious diarrhea following an alteration of normal intestinal flora by the use of broad-spectrum antimicrobials and leading to morbidity and mortality worldwide ${ }^{1}$. In 2011 , a surveillance study of CDI in U.S. identified the incidence of 453,000 cases with the estimated number of 29,300 deaths ${ }^{2}$. CDI has been ranked as a leading 
cause of death in people aged over 65 years in U.S. and Europe, but limited studies are undertaken elsewhere, particularly in $\mathrm{Asia}^{3,4}$. The prevalence of CDI in Thailand was $4.8-52.2 \%$ in diarrheal patients and 2.6-22.4\% in non-diarrheal patients, and mortality was up to $6.4 \%{ }^{4}$. From the 2010 nationwide data of Thailand, 554 patients in 570 admissions of 4.8 million hospital admissions were diagnosed with CDI (incidence rate $0.01 \%$ per admissions) and $19.1 \%$ died during the index hospitalization ${ }^{5}$. Currently, vancomycin or fidaxomicin have been the recommended antimicrobial treatment for CDI patient because they are better than metronidazole. The combination of oral vancomycin and metronidazole is also recommended for severe CDI with complication or critically ill patient ${ }^{6-9}$. However, fidaxomicin is not approved by Food and Drug Administration (FDA) of Thailand. As CDI patient was diagnosed each year but the burden of CDI and the rational use of antimicrobial therapy for CDI in our hospital was questionable. We present our experience regarding CDI in hospitalized patients over a three-year period in Srinagarind Hospital, a super tertiary care. Then, appropriateness and therapeutic outcome of antimicrobial therapy for CDI were also assessed.

\section{MATERIALS AND METHODS}

\subsection{Study design and patients}

This retrospective analysis was performed by reviewing patient medical records of hospitalized patients with CDI diagnosis at Srinagarind Hospital between October 2014 and September 2016. The inclusion criteria were patients more than 18 years of age and documented CDI diagnosis (ICD10-A047). CDI patients referred to other hospitals, presented with intra-abdominal complicated disorder including a perforated viscus or bowel obstruction or presented with recurrent CDI (CDI within 8 weeks of complete of therapy) or patients with missing or incomplete data were excluded.

CDI was defined as present of positive laboratory test for $C$. difficile toxin A or toxin B in the stool by chromatographic immunoassay using C.difficile toxin $\mathrm{A} / \mathrm{B}$ test kit (Remel Xpect $^{\circledR}$; Oxioid, UK). Community-associated CDI or hospital-onset CDI were defined as positive toxin test which presented in stool collected within or after 3 days of hospital admission; respectively, without overnight stay in a healthcare facility in the prior 12 weeks.
Asymptomatic carrier was patient with positive toxin test without any sign or symptoms. Symptomatic CDI was defined as present of diarrhea ( $\geq 3$ unformed stools in 24 hours) and positive toxin test. Severity of CDI was classified into 3 levels, mild to moderate disease (signs or symptoms not meeting severe or complicated criteria), severe disease (WBC $\geq 15,000$ cells $/ \mathrm{mm}^{3}$ or serum creatinine $>1.5 \mathrm{mg} / \mathrm{dL}$ ) and severe disease with complication (hypotension or shock, ileus, megacolon, end-organ failure) $)^{9}$.

\subsection{Data collection and analysis}

Eligible data were extracted from patient medical records included gender, age, health privilege, medical condition or comorbidity, infectious disease and treatment, sign and symptom of CDI and treatment, and total hospital stay. All data were reviewed for their completeness, and then appropriateness and therapeutic outcome of antimicrobial therapy for CDI were evaluated. The recommended medications were: mild to moderate CDI (vancomycin $125 \mathrm{mg}$ PO every $6 \mathrm{~h}$ or metronidazole $400 \mathrm{mg} \mathrm{PO} / 500 \mathrm{mg}$ IV every $8 \mathrm{~h}$ ), severe CDI (vancomycin $125 \mathrm{mg}$ PO every $6 \mathrm{~h}$ ) and severe CDI with complication (metronidazole $500 \mathrm{mg}$ IV every $8 \mathrm{~h}$ plus vancomycin $125-500$ $\mathrm{mg}$ PO every $6 \mathrm{~h}$ and/or rectal enema $500 \mathrm{mg}$ in $100-500 \mathrm{~mL}$ saline solution every $4-12 \mathrm{~h}$ and the duration of therapy should be 10-14 days ${ }^{6-9}$. Clinical outcome of CDI treatment was assessed from symptoms including fever or diarrhea. Clinical response was defined as resolution of diarrhea by day 7 and/or resolution of fever by day 3 of treatment ${ }^{7,10,11}$. Recovery rate was calculated as the number of patients with symptom resolution divided by the total patients with symptomatic CDI. Descriptive statistics were used to analyze the data. Discrete variables are presented as cumulative frequency and relative frequency $(\%)$ and continuous variables are expressed as median and range.

\section{RESULTS}

\subsection{Patient characteristics}

From 117,418 patient admissions and 793,669 patient-days, there were 237 patients with positive C.difficile toxin assay, but 69 patients were excluded. From the remaining 168 patients, male patients were slightly higher than female (57.7\% and $42.3 \%)$. The median age was 61 years 
(range 20-98 years). The median day before CDI diagnosed was 16 days (range 1-255 days) and the median length of total hospital stay was 30 days (range 3-362 days). Of 168 CDI patients, 113 (67.3\%) had at least one comorbidity which can contribute to the severity of illness. Cardiovascular disease was the most common comorbidity among CDI patients (Table 1).

\subsection{Infectious disease characteristics}

There were 176 episodes of infection disease diagnosed in 147 patients, but 141 patients received antimicrobials for their infections before CDI occurred (Table 2). Beta-lactams were the most commonly prescribed antimicrobials especially cephalosporins or carbapenems. The median duration from antimicrobial start to CDI appearance was 6 days (range 1-21 days). Totally, 27 patients did not receive any antimicrobial in the hospital prior to CDI presentation, 21 patients without other infectious disease and 6 patients without antimicrobial therapy for their infections.

The numbers of community-associated CDI and hospital-onset CDI were 27 and 141 cases, respectively; therefore, the calculated incidence rates were 0.23 cases $/ 1,000$ patient admissions and 1.78 cases/10,000 patient-days, respectively. Seventy-two percent of the patients presented symptomatic CDI, $41 \%$ with diarrhea and $31 \%$ with fever and diarrhea. Severity of
CDI was classified to mild to moderate, severe and severe with complicated disease in 55.9, 4.8 and $11.3 \%$ of CDI patients, respectively. Asymptomakitic carrier was accounted for $28 \%$ of the patients, $12 \%$ with fever and $16 \%$ with neither fever nor diarrhea. Metronidazole was the initial therapy in $83.9 \%$ of patients. Initial therapy with metronidazole, oral vancomycin and oral vancomycin plus metronidazole were prescribed in 141 (83.9\%), $8(4.8 \%)$ and 19 (11.3\%) patients, respectively. (Table 3) The median duration of treatment was 9 days (range 1-28 days).

\subsection{Medication use evaluation}

Appropriateness of CDI treatment was evaluated in terms of drug selection, dosage regimen and duration of treatment. Drug selection was appropriate and consistent with the severity of CDI in $72 \%$ of patients meanwhile inappropriate use was found in $28 \%$ of patients who were asymptomatic carrier. Dosage regimen of antimicrobial for CDI treatment was appropriate and consistent with the recommendation in $99.3 \%$ of patients. Duration of therapy in monophasic regimen was less than 10 days in 88 CDI patients and longer than 14 days in $6 \mathrm{CDI}$ patients. This account for inappropriate duration of therapy in $56 \%$ of all of all CDI treatment (Table 4).

Table 1. Patients' demographics.

\begin{tabular}{lc}
\hline Variable & Number of patient (\%) \\
\hline Gender & \\
\hline Male: Female & $97(57.7): 71(42.3)$ \\
\hline Age distribution & \\
\hline $18-35$ years & $15(8.9)$ \\
$36-50$ years & $31(18.5)$ \\
$51-65$ years & $50(29.8)$ \\
$65-80$ years & $54(32.1)$ \\
$>80$ years & $18(10.7)$ \\
\hline Health privilege & \\
\hline Universal coverage & $76(45.2)$ \\
Civil servant & $64(38.1)$ \\
Social security & $15(8.9)$ \\
Self-payment & $7(4.2)$ \\
Unknown & $6(3.6)$ \\
\hline Comorbidity & \\
\hline Cardiovascular disease & $80(47.6)$ \\
Diabetes & $46(27.4)$ \\
Chronic kidney disease & $35(20.8)$ \\
Cancer & $28(16.7)$ \\
Cerebrovascular disease & $19(11.3)$ \\
Human immunodeficiency virus infection & $16(9.5)$ \\
Chronic hepatitis & $6(3.6)$ \\
Chronic obstructive pulmonary disease & $4(2.4)$ \\
\hline
\end{tabular}


Table 2. Acute infectious diseases and antimicrobial exposures in CDI patients.

\begin{tabular}{lr}
\hline \multicolumn{1}{c}{ Variable } & $\begin{array}{c}\text { Number of patients } \\
\text { (\% of total patients) }\end{array}$ \\
\hline Infectious episodes during hospitalization $(\mathbf{n = 1 7 6 )}$ & $49(29.2)$ \\
\hline Respiratory tract & $20(11.9)$ \\
Bloodstream & $19(11.3)$ \\
Gastrointestinal & $15(8.9)$ \\
Urinary tract & $14(8.3)$ \\
Skin and soft tissue & $5(3.0)$ \\
Central nervous system & $33(48.2)$ \\
Multiple organs & $21(12.5)$ \\
None (prior to CDI diagnosed) & \\
\hline Patient received antimicrobial in hospital $(\mathbf{n = 1 6 8 )}$ & $60(35.7)$ \\
\hline Single agents & $81(48.2)$ \\
Multiple agents & $27(16.1)$ \\
None & \\
\hline Prescription containing antimicrobial $(\mathbf{n}=\mathbf{2 8 9})$ & $98(58.3)$ \\
\hline Beta-lactams & $61(36.3)$ \\
\hline Cephalosporins & $31(18.5)$ \\
Carbapenems & $3(1.8)$ \\
Betalactam/beta-lactamase inhibitors & $34(20.2)$ \\
Penicillins & $21(12.5)$ \\
Fluoroquinolones & $15(8.9)$ \\
Colistin & $10(6.0)$ \\
Cotrimoxazole & $8(4.8)$ \\
Clindamycin & $4(2.4)$ \\
Macrolides & $3(1.8)$ \\
Tetracyclines & $1(0.6)$ \\
Fosfomycin & \\
\hline Aminoglycosides & \\
\hline
\end{tabular}

Table 3. Characteristic and antimicrobial therapy of CDI.

\begin{tabular}{lr}
\hline \multicolumn{1}{c}{ Variable } & Number of patients $(\%)$ \\
\hline Acquisition & \\
\hline Community-associated CDI & $27(16.1)$ \\
Hospital-onset CDI & $141(83.9)$ \\
\hline Clinical presentation & $20(11.9)$ \\
\hline Fever & $69(41.1)$ \\
Diarrhea & $52(30.9)$ \\
Fever and diarrhea & $27(16.1)$ \\
None & \\
\hline Severity & $47(28.0)$ \\
\hline Asymptomatic carrier & $94(55.9)$ \\
Mild to moderate disease & $8(4.8)$ \\
Severe disease & $19(11.3)$ \\
Severe with complicated disease & \\
\hline Treatment & $85(50.6)$ \\
\hline Single course & $5(3.0)$ \\
Metronidazole & $13(7.7)$ \\
Vancomycin & \\
Combination & $29(17.3)$ \\
Sequential course & $16(9.5)$ \\
Metronidazole $\rightarrow$ vancomycin & $7(4.2)$ \\
Metronidazole $\rightarrow$ combination & $3(1.7)$ \\
Metronidazole $\rightarrow$ combination $\rightarrow$ vancomycin & $1(0.6)$ \\
Metronidazole $\rightarrow$ combination $\rightarrow$ metronidazole & $1(0.6)$ \\
Metronidazole $\rightarrow$ vancomycin $\rightarrow$ combination & $2(1.2)$ \\
Vancomycin $\rightarrow$ metronidazole & $2(1.2)$ \\
Vancomycin $\rightarrow$ combination $\rightarrow$ vancomycin & $4(2.4)$ \\
Combination $\rightarrow$ metronidazole & \\
Combination $\rightarrow$ vancomycin & \\
\hline
\end{tabular}


Table 4. Appropriateness of antimicrobial therapy for CDI.

\begin{tabular}{|c|c|c|}
\hline \multirow{2}{*}{ Categories } & \multicolumn{2}{|c|}{ Appropriateness (\%) } \\
\hline & Yes & No \\
\hline \multicolumn{3}{|l|}{ Drug selection $(n=168)$} \\
\hline Metronidazole & $94(55.9)$ & $47(28.0)$ \\
\hline Vancomycin & $8(4.8)$ & - \\
\hline Metronidazole plus vancomycin and/or vancomycin PR & $19(11.3)$ & - \\
\hline \multicolumn{3}{|l|}{ Dosage regimen $(n=297)$} \\
\hline Metronidazole PO $400 \mathrm{mg}$ every $8 \mathrm{~h}$ & $123(41.4)$ & - \\
\hline Metronidazole IV $500 \mathrm{mg}$ every $8 \mathrm{~h}$ & $74(24.9)$ & - \\
\hline Vancomycin PO $125 \mathrm{mg}$ every $6 \mathrm{~h}$ & $50(16.8)$ & - \\
\hline Vancomycin PO $250 \mathrm{mg}$ every $6 \mathrm{~h}$ & $19(6.4)$ & - \\
\hline Vancomycin PO 500 mg every $6 \mathrm{~h}$ & $22(7.4)$ & - \\
\hline Vancomycin enema $500 \mathrm{mg}$ in saline solution every $4-12 \mathrm{~h}$ & $7 \quad(2.4)$ & - \\
\hline Other & - & $2(0.7)$ \\
\hline \multicolumn{3}{|l|}{ Duration $(n=168)$} \\
\hline less than 10 day & - & $88(52.4)$ \\
\hline $10-14$ days & $57(33.9)$ & - \\
\hline more than 14 days & $17(10.1)$ & $6 \quad(3.6)$ \\
\hline
\end{tabular}

Finally, appropriate CDI treatment in all 3 aspects (drug selection, dosage regimen and duration) were founded in 74 patients (44\%).

In term of clinical outcome, symptom disappear was achieved in 75 of 121 patients with symptomatic CDI, 28 of 69 diarrheal patients without fever and 47 of 52 diarrhea patients with fever. Therefore, the calculated recovery rate was $62 \%$. Nine patients died during CDI treatment.

\section{DISCUSSION}

This is the first study reported both the burden of CDI and medication use evaluation for CDI treatment in Thailand. The incidence rate of CDI in our hospital ( 0.23 case $/ 1,000$ patient admissions) was higher than the 2010 nationwide CDI incidence of Thailand $(0.01 \%$ per admission or 0.1 case/1000 patient admissions) $)^{5}$. The data also supported that the incidence of CDI in Eastern countries was lower than in Western countries. Difference in diet and gut microbiota among regional population may be important protective factors associated with CDI. However, the incidence rate of CDI patient in this study may be underestimated due to stool testing for CDI is not routinely performing in all patients with diarrhea in our hospital. In this study, the diagnosis of CDI using a clinical definition of diarrhea and the detection of C.difficile toxin $\mathrm{A} / \mathrm{B}$ in the stool by immunochromatography has sensitivity and specificity of $86.3 \%$ and $96.2 \%$, respectively. This test has been adopted by many hospital laboratories because it is a rapid test and provides excellent clinical performance with a simple, easy-to-use procedure and rapid report.
In contrast, fecal leukocyte was not evaluated in our patients because this test has poor sensitivity and is not a good screening test for CDI.

Our data are consistent with the previous epidemiological study that old age, co-morbidity, antimicrobial exposure, and prolonged hospital stay are important risk factors for $\mathrm{CDI}^{1,11}$. Although the median age of CDI patients in this study was 61 years, $42.8 \%$ of CDI patients was older than 65 years. Elderly population usually presented with co-morbidity which compromised the host immunity ${ }^{12}$. Co-morbidities commonly noted in our study were cardiovascular disease, diabetes mellitus, chronic kidney disease and malignancy and some patients had many comorbidities similar to previous studies ${ }^{5,13}$. The median duration of admission and the median duration of antibiotic exposure before developing positive toxin test were 16 days and 6 days, respectively. Most of patients (83.9\%) had received antimicrobial prior to CDI presentation similar to study by Thipmontree et al. ${ }^{13}$ Use of broad-spectrum antimicrobial or antimicrobial combination may alter normal flora in gastrointestinal tract, cause overgrowth of C.difficile and then contribute to CDI. The most common exposed antimicrobial was beta-lactam antibiotic either alone or in combination similar to study by Ngamskulrungroj et al. ${ }^{14}$ Our patients received cephalosporins (58.3\%), carbapenems (36.3\%) and/or fluoroquinolone (20.2\%) that reflected the pattern of antibiotic use in the hospital rather than the direct relationship between specific antimicrobials to CDI since long term exposure to antibiotic play a more significant risk for CDI rather than short course administration or type of 
antibiotic $^{15}$. However, the CDI risk and appropriate use of these antibiotics were not assessed in this study. In addition, this retrospective study did not capture the history of outpatient antibiotic use, history of previous admission from other causes and other possible associated risk factors such as nonsteroidal anti-inflammatory drugs (NSAIDs), proton-pump inhibitors (PPIs) prescription ${ }^{16,17}$.

In the previous guideline, metronidazole was recommended as initial therapy for CDI patients with mild to moderate symptoms and vancomycin was recommended in severe cases or CDI with complicated, fulminant or recurrent CDI, meanwhile, either vancomycin or fidaxomicin is recommended rather than metronidazole for an initial episode for CDI in the current guideline $^{6-9}$. Previous study in a large tertiary care teaching hospital in U.S. reported that metronidazole was the most commonly used drug $(81.6 \%)$ for CDI treatment. ${ }^{18}$ Study in a French university hospital also reported metronidazole alone was used in $73.5 \%$ for CDI treatment despite severe CDI was observed in $53.9 \%$ of all CDI patients. ${ }^{19}$ In our study, metronidazole was started in $83.9 \%$ of CDI cases because most patients had non-severe CDI. However, inappropriate medication in drug selection occurred in $28 \%$ of CDI patients because antimicrobial therapy has not been recommended for patients with asymptomatic CDI. Although, asymptomatic carriers of $C$. difficile were more likely to develop difficult to treat diarrhea with a significantly high death rate or may be transferred to other patients, the CDI guideline proposes 3 suggestions; screening in order to prevent development of diarrhea (e.g. by providing probiotics), initiating CDI treatment early if suggestive symptoms occur, and preventing transmission of $C$. difficile (by implementing isolation measures) $)^{17,20}$. Beyond giving antimicrobial to treat asymptomatic carriers, inappropriate duration of therapy ( $<10$ days) was seen in patient with drug discontinuation before treatment complete. Therefore, pharmacist should intervene the doctor to complete the treatment regimen. This may help in decrease an occurrence of recurrent CDI. On the other hand, too long duration of treatment (> 2 weeks) was found in single course in which automatic stop order policy may prevent unnecessarily prolonged therapy and encourage reassessment of need to change therapy. The combination of metronidazole and vancomycin was given in severe CDI with complicated disease for higher efficacy and less mortality ${ }^{21-23}$. This combination also used in sequential course with duration over 14 days for patient with unresponsiveness to initial treatment. However, the appropriate duration of treatment in this situation is still in question and need for further investigation. Recovery rate of patients in this study was $62 \%$ which was similar to previous study that reported clinical cure rate of 53.7 to 98\% depend on the treatment regimen and severity of $\mathrm{CDI}^{8-10,22,23}$.

In term of drug formulation, metronidazole tablet (200 and $400 \mathrm{mg}$ ) and metronidazole sterile solution for injection (500 $\mathrm{mg} / 10 \mathrm{~mL}$ ) are available but oral vancomycin capsule is not available in Thailand. Oral metronidazole $400 \mathrm{mg}$ is selected for treating mild to moderate CDI and IV metronidazole $500 \mathrm{mg}$ is used in patients who cannot take the drug by mouth as proposed by Public Health England ${ }^{7}$. Vancomycin powder for injection is given orally in severe CDI since it has minimal absorption from the gastrointestinal tract to blood circulation. By the way, another applicable route of vancomycin is $500 \mathrm{mg}$ in NSS $500 \mathrm{ml}$ given as rectal enema until the patient has significant clinical improvement.

The main limitations of our study are secondary to retrospective data collection. All information was obtained from patient medical records; therefore, the information was limited. Details regarding the stage of chronic kidney disease and glycemic control, stage and type of cancer and chemotherapeutic agent were not extracted in detail. The reason for the antibiotic prescription prior to CDI and the number of readmissions were also not evaluated. However, the finding of our study can represent the actual situation and guide health policy decision in the future to improve rational drug use in hospital.

\section{CONCLUSIONS}

The incidence rate of CDI patients at Srinagarind Hospital was relatively low but may be underestimated. Most patients had mild to moderate CDI and was initially treated with metronidazole $(83.9 \%)$ that should change to use oral vancomycin as the first-line therapy. Appropriate treatment was found in $44.0 \%$ of CDI patients and recovery rate was $62.0 \%$ Antimicrobial therapy in asymptomatic carrier was the major cause of inappropriate medication that must be diminished. Other hospital policies or strategies should be proposed to prevent CDI and promote rational drug use. 
Conflict of interest (If any)

The authors declare no conflict of interests.

\section{Funding \\ None to declare}

\section{Ethical approval}

The study was approved by the Khon Kaen University Ethics Committee for Human Research based on the Declaration of Helsinki and ICH Good Clinical Practice Guidelines (HE591552).

\section{Article info:}

Received June 12, 2020

Received in revised form July 24, 2020

Accepted November 13, 2020

\section{REFERENCES}

1. Leffler DA, Lamont T. Clostridium difficile infection. $\mathrm{N}$ Engl J Med. 2015;372(16):1539-48.

2. Lessa FC, Mu Y, Bamberg WM, Beldavs ZG, Dumyati GK, Dunn JR, et al. Burden of Clostridium difficile infection in the United States. N Engl J Med. 2015;372(9) :825-34.

3. Wilson R, Beerbaum P, Giglio S. Community and hospital acquired Clostridium difficile in South Australia - ribotyping of isolates and a comparison of laboratory detection methods. Lett Appl Microbiol. 2015;60(1):33-6.

4. Putsathit P, Kiratisin P, Ngamwongsatit P, Riley TV. Clostridium difficile infection in Thailand. Int $\mathrm{J}$ Antimicrob Agents. 2015;45(1):1-7.

5. Pittayanon R, Treeprasertsuk S, Phaosawasdi K, Kitsahawong B, Thepsuthammarat K, Kullavanijaya P. The burden of Clostridium difficile infection in Thais: nationwide survey. Asian Biomed. 2015;9(6):738-8.

6. Cohen SH, Gerding DN, Johnson S, Kelly CP, Loo VG, McDonald LC, et al. Clinical Practice Guidelines for Clostridium difficile Infection in Adults: 2010 update by the Society for Healthcare Epidemiology of America (SHEA) and the Infectious Diseases Society of America (IDSA). Infect Control Hosp Epidemiol. 2010;31(5):431-55.

7. Public Health England. Updated guidance on the management and treatment of Clostridium difficile infection [document on the internet]. London; 2013. [cited 2020 Jun 30]. Available from: https://assets. publishing.service.gov.uk/government/uploads/system/ uploads/attachment_data/file/321891/Clostridium_difficile_management_and_treatment.pdf

8. Debast SB, Bauerb MP, Kuijpercon EJ. European Society of Clinical Microbiology and Infectious Diseases: update of the treatment guidance document for Clostridium difficile infection. Clin Microbiol Infect. 2014;20 (Suppl. 2):1-26.

9. McDonald LC, Gerding DN, Johnson S, Bakken JS, Carroll KC, Coffin SE, et al. Clinical practice guidelines for Clostridium difficile infection in adults and children: 2017 Update by the Infectious Diseases Society of
America (IDSA) and Society for Healthcare Epidemiology of America (SHEA). Clin Infect Dis. 2018; 66(7):e1-e4.

10. Zar FA, Bakkanagari SR, Moorthi KM, Davis MB. A comparison of vancomycin and metronidazole for the treatment of Clostridium difficile-associated diarrhea, stratified by disease severity. Clin Infect Dis. 2007;45(3):302-7.

11. Schroeder MS. Clostridium difficile-Associated Diarrhea. Am Fam Physician 2005;71(5):921-8.

12. Daniel A, Rapose A. The evaluation of Clostridium difficile infection (CDI) in a community hospital. J Infection Public Health. 2015;8:155-60.

13. Thipmontree W, Kiratisin $\mathrm{P}$, Manatsathit S, Thamlikitkul V. Epidemiology of suspected Clostridium difficile-associated hospital-acquired diarrhea in hospitalized patients at Siriraj Hospital. J Med Assoc Thai. 2011;94 (Suppl 1):S207-16.

14. Ngamskulrungroj P, Sanmee S, Pusathit P, Piewngam $\mathrm{P}$, Elliott B, Riley TV, et al. Molecular epidemiology of Clostridium difficile infection in a large teaching hospital in Thailand. PLoS One. 2015;10(5):e0127026.

15. Steven V, Dumyati F, Fine LS, Fisher SG, van Wijngaarden E. Cumulative antibiotic exposures over time and the risk of Clostridium difficile infection. Clin Infect Dis. 2011;53(1):42-8.

16. Manges AR, Labbe A, Loo VG, Atherton JK, Behr MA, Masson L, et al. Comparative metagenomic study of alterations to the intestinal microbiota and risk of nosocomial Clostridium difficile-associated disease. J Infect Dis. 2010;202(12):1877-84.

17. Dials S, Alrasadi K, Manoukian C, Huang A, Menzies D. Risk of Clostridium difficile diarrhea among hospital inpatients prescribed proton pump inhibitors: cohort and case-control studies. CMAJ. 2004:171:33-8.

18. Salazar M, Garey KW, Jiang ZD, Dao-Tran T, DuPont H. Changing Clostridium difficile infection testing and treatment trends at a large tertiary care teaching hospital. Pharm World Sci. 2009;31(5):565.

19. Khanafer N, Blais L, Barbut F, Hirschel B, Vanhems P. Treatment of Clostridium difficile infection in a French university hospital. Scand J Gastroenterol. 2015;50(10): 1253-60.

20. Kagan S, Wiener-Wella Y, Ben-Chetrit E, Kashat L, Aouizerats $\mathrm{J}$, Bdolah-Abram $\mathrm{T}$, et al. The risk for Clostridium difficile colitis during hospitalization in asymptomatic carriers. J Hosp Infect. 2017;95(4):442-3.

21. Dubberke ER, Carling P, Carrico R, Donskey CJ, Loo VG, McDonald LC, et al. Strategies to prevent Clostridium difficile infections in acute care hospitals: 2014 update. Infect Control Hosp Epidemiol. 2014;35(6): 628-45.

22. Rokas KEE, Johnson JW, Beardsley JR, Ohl CA, Luther VP, Williamson JC. The addition of intravenous metronidazole to oral vancomycin is associated with improved mortality in critically ill patients with Clostridium difficile infection. Clin Infect Dis. 2015;61 (6):934-41.

23. Li R, Lu L, Lin Y, Wang M, Liu X. Efficacy and safety of metronidazole monotherapy versus vancomycin monotherapy or combination therapy in patients with Clostridium difficile infection: A systematic review and meta-analysis. PLoS ONE 2015;10(10): e0137252. 